\title{
STRUCTURE OF LINEAR SETS
}

\author{
BY \\ ERNST SNAPPER
}

\section{INTRODUCTION}

In general only vector spaces whose scalar domains are fields have been investigated. In this paper we study vector spaces whose scalar domains are integral domains in which every ideal has a finite basis. It is shown that the theory of the linear subsets of such a vector space can be developed by using exactly the same technique which has always been applied to the ideals of an integral domain. This technique is established in the first two sections and is then used to develop the Noether decomposition theory of linear sets into "primary" linear sets.

\section{BASIS THEOREM AND ASCENDING CHAIN CONDITION}

We first define the notion of vector space:

Definition 1.1. An n-dimensional vector space consists of all the vectors with $n$ components where the components form an integral domain in which every ideal has a finite basis.

This integral domain is called the scalar domain of the vector space.

Definition 1.2. A linear set is a subset of an $n$-dimensional vector space closed under vector subtraction and multiplication by arbitrary scalars.

An integral domain in which every ideal has a finite basis is clearly a onedimensional vector space and its ideals are the linear sets. The ordinary theory of ideals will therefore become a part of this theory of linear sets.

Notation. The fixed $n$-dimensional vector space will be indicated by $V_{n}$ and its scalar domain by $R$. Otherwise the capital Latin letters will be used exclusively to indicate linear sets and the small Latin letters to indicate vectors. The ideals of $R$ will be indicated by German letters and the scalars of $R$ by Greek letters. The radical of an ideal $\mathfrak{a}$ will be indicated by $\mathfrak{a}^{\prime}$. As is customary, $\mathfrak{a}^{\prime}$ consists of all the scalars of which a power lies in $\mathfrak{a}$.

The following theorems, whose proofs are apparent from van der Waerden's Moderne Algebra, vol. 2 (we refer to this book as [1]), will be used frequently:

THEOREM 1.1 (The basis theorem). Every linear set has a finite basis.

Presented to the Society, February 22, 1941; received by the editors December 3, 1940, and, in revised form August 9, 1941. 
THEOREM 1.2 (The ascending chain condition). A chain of linear sets $L_{1} \subset L_{2} \subset \cdots$ in which $L_{i}$ is properly contained in $L_{i+1}$ is finite.

THEOREM 1.3 (Maximum condition). Every non-empty set of linear sets possesses a maximal set (that is, a linear set which is not contained in any other linear set of that set).

THEOREM 1.4 (Induction by division). If a property can be proved for every linear set $L, V_{n}$ included, under the assumption that the property can be proved for all the linear sets which properly contain $L$, the property holds for every linear set.

\section{Products AND QuOtients}

The technique mentioned above for treating the linear sets is based upon the following definitions:

Definition 2.1. The greatest common divisor or sum $L=\left(L_{1}+L_{2}\right)$ of two linear sets $L_{1}$ and $L_{2}$ is the linear set $L$, generated by their logical sum.

Definition 2.2. The least common multiple $L=\left[L_{1} \cap L_{2}\right]$ of two linear sets $L_{1}$ and $L_{2}$ is their intersection.

Definition 2.3. The product $L=\mathfrak{a} L_{1}$ of an ideal $\mathfrak{a}$ and a linear set $L_{1}$ is the linear set $L$, generated by the scalar products of scalars of a and vectors of $L_{1}$.

Definition 2.4. The quotient $L=L_{1} / \mathfrak{a}$ of a linear set $L_{1}$ and an ideal $\mathfrak{a}$ is the linear set $L$, consisting of the vectors whose scalar products with all the scalars of a lie in $L_{1}$.

Definition 2.5. The quotient $\mathfrak{a}=L_{1} / L_{2}$ of a linear set $L_{1}$ and a linear set $L_{2}$ is the ideal $\mathfrak{a}$, consisting of the scalars whose scalar products with all the vectors of $L_{2}$ lie in $L_{1}$.

The theorems concerning these notions are the same as in the case of ideals and are proved in the same way. The most important ones are listed below (for notation see Part I):

THEOREM 2.1. Multiplication is associative and is distributive with respect to addition:

$\left(\mathfrak{a}_{1} \mathfrak{a}_{2}\right) L=\mathfrak{a}_{1}\left(\mathfrak{a}_{2} L\right) ; \quad\left(\mathfrak{a}_{1}+\mathfrak{a}_{2}\right) L=\left(\mathfrak{a}_{1} L+\mathfrak{a}_{2} L\right) ; \quad \mathfrak{a}\left(L_{1}+L_{2}\right)=\left(\mathfrak{a} L_{1}+\mathfrak{a} L_{2}\right)$.

THEOREM 2.2. Division is distributive with respect to intersection:

$$
\begin{aligned}
{\left[L_{1} \cap L_{2} \cap \cdots \cap L_{r}\right] / M } & =\left[L_{1} / M \cap L_{2} / M \cap \cdots \cap L_{r} / M\right] ; \\
{\left[L_{1} \cap L_{2} \cap \cdots \cap L_{r}\right] / \mathfrak{a} } & =\left[L_{1} / \mathfrak{a} \cap \cdots \cap L_{r} / \mathfrak{a}\right] .
\end{aligned}
$$

THEOREM 2.3. The quotient of the sum is the intersection of the quotients:

$$
L /\left(\mathfrak{a}_{1},+\mathfrak{a}_{2}\right)=\left[L / \mathfrak{a}_{1} \cap L / \mathfrak{a}_{2}\right] ; \quad L /\left(M_{1}+M_{2}\right)=\left[L / M_{1} \cap L / M_{2}\right] .
$$




\section{Closure, essential ideal and Radical}

The notions discussed in this section play an important role in the structure theory of linear sets.

Definition 3.1. The closure $\bar{L}$ of a linear set $L$ is the linear set consisting of all the vectors of which some scalar multiple, different from zero, lies in $L$.

This definition is clearly consistent and we always have

$$
L \subseteq \bar{L}, \quad \overline{\left.L_{1} \cap L_{2}\right]}=\left[\bar{L}_{1} \cap \bar{L}_{2}\right]
$$

Definition 3.2. The linear set $L$ will be called closed if $\bar{L}=L$ and dense if $\bar{L}=V_{n}$

EXAMPLE 3.1. If $R$ consists of all the rational integers, then in $V_{2}$ over $R$ the set $L_{1}$ generated by $(2,2)$ is neither closed nor dense; $L_{2}=(1,1)$ is closed but not dense; $L_{3}$, the set generated by $(1,0)$ and $(0,2)$, is not closed but is dense.

Every closure of a linear set is a closed set and the nonzero ideals, considered as the linear subsets of a one-dimensional vector space, are dense sets. The only linear set which is both closed and dense is $V_{n}$.

The following theorem will be used later:

THEOREM 3.1. The intersection of two closed sets is closed. The intersection of two dense sets is dense. The irredundant intersection of a closed set and a dense set is neither closed nor dense. (An intersection is called irredundant if none of the intersection components is superfluous.)

This theorem follows immediately from the fact that

$$
\overline{\left[L_{1} \cap L_{2}\right]}=\left[\bar{L}_{1} \cap \bar{L}_{2}\right] \text {. }
$$

Definition 3.3. The essential ideal $\mathbb{E}=L / V_{n}$ of a linear set $L$ is the quotient ideal of the linear set and the whole space $V_{n}$. The radical ${ }^{\prime}$ of $L$ is the radical of $\leftleftarrows$.

The essential ideal $\&$ clearly consists of all the scalars which transform the whole space by means of scalar multiplication into the linear set. Therefore we have $L / \&=V_{n}$. The essential ideal of an ideal is the ideal itself and the radical of an ideal is just its ordinary radical.

THEOREM 3.2. The following three statements are equivalent: $L$ is dense; $\mathbb{E} \neq 0 ; L$ has maximal dimension $n$.

Proof. If $L$ is dense, the ideals $L /(1,0, \cdots, 0), L /(0,1,0, \cdots, 0), \cdots$, $L /(0, \cdots, 1)$ are nonzero ideals and therefore $\xi=L / V_{n}=[L /(1,0, \cdots, 0)$ $\cap \cdots \cap L /(0, \cdots, 1)]$ is a nonzero ideal. If $\mathbb{E} \neq 0$, there exists a scalar $\lambda$ such that the $n$ vectors $(\lambda, 0, \cdots, 0) \cdots(0, \cdots, \lambda)$ lie in $L$ and therefore $L$ 
has maximal dimension $n$. If $L$ has maximal dimension $n$, there are $n$ independent vectors $f_{1}, \therefore, f_{n}$ in $L$. An arbitrary vector $v$ may then be written as $v=\left(\alpha_{1} / \beta_{1}\right) f_{1}+\cdots+\left(\alpha_{n} / \beta_{n}\right) f_{n}$ and therefore $\lambda v=\rho_{1} f_{1}+\cdots+\rho_{n} f_{n}$ lies in $L$. Consequently, $L$ is dense. This proves the theorem.

We conclude this section by remarking that $L \subseteq L / \mathfrak{a} \subseteq \bar{L}$ and $\xi_{L} \subseteq L / M \subseteq R$, where $\mathfrak{a}$ has to be a nonzero ideal.

\section{PRIMARY LINEAR SETS AND PRIME LINEAR SETS}

Definition 4.1. A linear set $L$ will be called primary if $\lambda v \equiv 0(L)$ implies either $v \equiv 0(L)$ or $\lambda \equiv 0\left(\mathbb{F}^{\prime}\right)$.

Definrtion 4.2. A linear set will be called prime if the linear set is primary and its (F) is a prime ideal.

It is clear that the ideals which are prime or primary according to these definitions are the ordinary prime and primary ideals.

THEOREM 4.1. If $L$ is primary $\&$ is primary and therefore $\xi^{\prime}$ is prime.

Proof. $\alpha \beta \equiv 0$ (ङ) and $\beta \not \equiv 0$ (ङ) imply $L / \alpha \beta=V_{n}$ and $L / \beta \neq V_{n}$. We can find therefore a vector $v$ such that $\alpha \beta v \equiv 0(L)$ and $\beta v \not \equiv(L)$ from which it follows that $\alpha \equiv 0\left(\&^{\prime}\right)$, q.e.d.

The converse does not hold, since the set generated by the vector $(2,4)$ in the vector space whose scalar domain consists of the rational integers is not primary while its essential ideal is the zero ideal and therefore prime.

Theorem 4.2. A primary linear set is either closed or dense.

Proof. If $\leftleftarrows \neq 0, L$ is dense. If $\mathbb{E}=0, \mathbb{E}^{\prime}=0$, from which we conclude that if $\lambda v \equiv 0(L)$ and $v \not \equiv 0(L), \lambda$ has to be zero. This means that $L$ is closed.

The converse does not hold since an ideal is a dense set but may not be primary. However, every closed set is a prime linear set since every closed linear set other than $V_{n}$ has a zero essential ideal.

The following theorems will demonstrate further that a primary linear set is the exact analogue of a primary ideal. These theorems are proved in exactly the same way as in the case of ideals and are used constantly in the structural theory of linear sets.

THEOREM 4.3. Let $L$ be an arbitrary linear set, $\$$ its essential ideal and $\mathfrak{a}$ an arbitrary ideal. Then $L$ is primary and $\mathfrak{a}$ is equal to the radical (ङ' of the linear set if and only if the following three conditions hold: (1) $\lambda v \equiv 0(L)$ implies either $v \equiv 0(L)$ or $\lambda \equiv 0(\mathfrak{a}) ;(2)\left(\xi \subseteq \mathfrak{a} ;(3) \lambda \equiv 0(\mathfrak{a})\right.$ implies $\lambda^{k} \equiv 0(\mathbb{E})$ for some $k$.

THEOREM 4.4. Let $L$ be a primary linear set, $\mathfrak{a}$ an ideal and $M$ a linear set. Then $\mathfrak{a} M \equiv 0(L)$ implies either $M \equiv 0(L)$ or $\mathfrak{a} \equiv 0\left(\&^{\prime}\right)$.

CoRollary 4.1. If $L$ is primary and $\mathfrak{a} \neq \equiv\left(\mathfrak{f}^{\prime}\right), L / \mathfrak{a}=L$. 
THEOREM 4.5. If $L=\left[L_{1} \cap L_{2} \cap \cdots \cap L_{r}\right]$, we have $\mathbb{F}=\left[\xi_{1} \cap \xi_{2} \cap \cdots \cap \xi_{r}\right]$ and $\mathfrak{F}^{\prime}=\left[\xi_{1}^{\prime} \cap \xi_{2}^{\prime} \cap \cdots \cap \xi_{r}^{\prime}\right]$, where $\mathfrak{F}_{i}$ and $\mathfrak{F}_{i}^{\prime}$ are the essential ideal and radical of $L_{i}$.

\section{THE NOETHER DECOMPOSITION THEORY}

A linear set will of course be called reducible if it is the irredundant intersection of two other linear sets and irreducible if it is not reducible.

From Theorem 1.2, the following theorem is derived:

THEOREM 5.1. Every linear set is the irredundant intersection of a finite number of irreducible linear sets.

After each of the following theorems we have indicated how to derive the proofs of these theorems from the very similar proofs of the corresponding theorems on ideals, given in [1].

THEOREM 5.2. Every irreducible linear set is primary.

Proof. (See [1, pp. 31 and 32].) Replace the radical of the ideal by the radical of the linear set and the integral domain by the vector space.

An immediate corollary of Theorems 5.1 and 5.2 is:

THEOREM 5.3. Every linear set is the irredundant intersection of a finite number of primary linear sets.

THEOREM 5.4. The intersection of a finite number of primary linear sets which all have the same radical is a primary linear set with that same radical.

Proof. (See [1, pp. 32 and 33].) Use Theorem 4.3.

By replacing the intersection components which have the same radical by their intersection we prove:

THEOREM 5.5. Every linear set is the irredundant intersection of a finite number of primary linear sets whose radicals are all different.

A representation of a linear set as described in Theorem 5.5 is called "a representation by maximal primary components."

As a preparation for the first uniqueness theorem we need the following lemma:

LеммA 5.1. In two representations of a linear set by maximal primary components, each radical which is maximal among all the radicals of both the representations is present in both the representations.

Proof. (See [1, pp. 35, 36].) Divide the components of both the representations by the essential ideal of a primary component whose radical is maximal among all the radicals of both the representations. Use Corollary 4.1.

From this lemma the following theorem is easily derived: 
Theorem 5.6. An irredundant intersection of a finite number of primary linear sets, whose radicals are not all the same, is not primary.

This theorem shows that in a representation of a linear set by maximal primary components no group of components has a primary intersection.

THEOREM 5.7 (The first uniqueness theorem). In two representations of a linear set by maximal primary components, the number of components is the same and so are the radicals of the components of the two representations.

Proof. (See [1, p. 36].) Single out, from the two representations, two linear sets $L_{1}$ and $L_{2}$ whose radicals $\xi_{1}^{\prime}$ and $\xi_{2}^{\prime}$ are the same and are maximal among all the radicals of both the representations. Then divide both the representations by the intersection of the two essential ideals of $L_{1}$ and $L_{2}$.

The uniquely determined radicals which occur in a representation of a linear set by maximal primary components are prime ideals (Theorem 4.1). They will be called the "associated prime ideals" of the linear set.

The following theorems are essential for the proof of the second uniqueness theorem:

TheOREM 5.8. We have $M / \mathbb{E}_{L}=M$ if and only if the essential ideal $\mathfrak{E}_{L}$ of the linear set $L$ is not contained in any of the associated prime ideals of the linear set $M$.

Proof. (See [1, p. 37].) Divide by $\mathcal{E}_{L}$ and use the fact that $\mathcal{E}_{1} N_{2} \subseteq\left[N_{1} \cap N_{2}\right]$, where $N_{1}$ and $N_{2}$ are linear sets and $\mathbb{E}_{1}$ is the essential ideal of $N_{1}$.

Another way of stating this theorem is:

THEOREM 5.9. We have $M / \mathfrak{E}_{L}=M$, where $\xi_{L}$ is the essential ideal of $L$, if and only if no associated prime ideal of $L$ is contained in an associated prime ideal of $M$.

This theorem leads to:

DEFINITION 5.1. The linear set $L$ is relatively prime with respect to the linear set $M$ if and only if $M / \mathfrak{\xi}_{L}=M$.

The following definitions lead to the second uniqueness theorem:

Definition 5.2. An associated prime ideal of a linear set is said to be imbedded if it contains another associated prime ideal of that linear set.

Definition 5.3. An intersection of maximal primary components, all belonging to the same representation of a linear set $L$ by maximal primary components, is called a component linear set of $L$.

DEFINITION 5.4. Two component linear sets, which are taken from the same representation of a linear set $L$ by maximal primary components and which have intersection $L$, are called conjugate component linear sets of $L$. 
If we call the conjugate of $L$, considered as a component linear set of itself, $V_{n}$, we have: Every component linear set of a linear set has a conjugate, which is not necessarily unique.

Definition 5.5. A component linear set of a linear set is said to be isolated if none of its associated prime ideals contains an associated prime ideal of its conjugate.

Theorem 5.10 (The second uniqueness theorem). An isolated component linear set of a linear set is uniquely determined by its associated prime ideals.

Proof. (See [1, p. 38].) Use Theorem 5.9. If in [1] the author divides by an ideal, here we have to divide by the essential ideal of the corresponding linear set.

\section{TWO CONSEQUENCES OF THE STRUCTURE THEORY}

It might be possible that the associated prime ideals of a linear set were always the same as the associated prime ideals of the essential ideal of that linear set. This would suggest that the representation of the linear set by maximal primary components were induced by the Noether decomposition of the essential ideal of the linear set. However, this conjecture will be disproved by the following counterexample:

EXAMPLE 6.1. Let $V_{2}$ have the polynomials in two variables $x$ and $y$ with integral coefficients as scalar domain. Let $L_{1}$ be the linear set consisting of all the vectors whose two components have a difference congruent to zero modulo $\left(x^{2}\right)$, and $L_{2}$ the linear set consisting of the vectors whose two components have a sum congruent to zero modulo $\left(x^{2}, y\right)$. Consider finally the linear set $L=\left[L_{1} \cap L_{2}\right]$. The following statements can easily be verified:

$L_{1}$ is a primary linear set. $\xi_{1}=\left(x^{2}\right)$ and $\xi_{1}^{\prime}=(x)$.

$L_{2}$ is a primary linear set. $\xi_{2}=\left(x^{2}, y\right)$ and $\xi_{2}^{\prime}=(x, y)$.

$L=\left[L_{1} \cap L_{2}\right]$ is a representation of $L$ by maximal primary components. The associated prime ideals of $L$ are $(x)$ and $(x, y)$. However, the essential ideal of $L$ is $\left(x^{2}\right)$ and its only associated prime ideal is $(x)$.

This example illustrates the general situation described in the following theorem:

ThEOREM 6.1. The associated prime ideals of the essential ideal of a linear set are among the associated prime ideals of that linear set.

Proof. From Theorem 4.5 we see that the representation of a linear set by maximal primary components induces a decomposition of the essential ideal into primary ideals, whose radicals are all different and equal to the associated primes of the linear set. However, this decomposition may not be irredundant, as Example 6.1 shows, where this induced decomposition is $\left(x^{2}\right)=\left[\left(x^{2}\right) \cap\left(x^{2}, y\right)\right]$. By deleting the unnecessary components in the inter- 
section, we get the Noether decomposition of the essential ideal and the remaining radicals are the associated primes of the essential ideal.

THEOREM 6.2. Every linear set is the irredundant intersection of a closed set and a dense set. The closed part of the intersection is unique and is the closure of the set. The dense part of the intersection is not unique, even when the intersections are restricted to representations by maximal primary components. The dense part of the intersection is not present if and only if the linear set is closed, and the closed part of the intersection is not present if and only if the set is dense.

Proof. Consider a representation of the linear set by maximal primary components. From Theorems 3.1 and 3.2 it follows that the intersection of the intersection components whose radicals differ from zero is a dense set and that the intersection component whose radical is the zero ideal is a closed set. This dense set and this closed set clearly satisfy the requirements of the theorem. That the closed part of the intersection is the closure of the linear set is proved as follows: Let $L=[C \cap D]$ where $D$ is dense and $C$ is closed. Then $\bar{L}=[\bar{C} \cap \bar{D}]$ (Part III) and $\bar{C}=C$ and $\bar{D}=V_{n}$. Therefore $\bar{L}=C$. The last part of the theorem follows immediately from Theorem 3.1. Finally, the following example shows that the dense part of the intersection is not unique, even if we restrict ourselves to representations by maximal primary components:

EXAMPLE 6.2. Let $V_{2}$ have the rational integers as scalar domain. Let $L$ be the linear set generated by the vector $(2,2)$. The following statements can easily be verified: The linear set generated by the vector $(1,1)$ is the closure $\bar{L}$ of $L . L$ is the intersection of $\bar{L}$ and the dense set generated by the vectors $(2,2)$ and $(1,3) . L$ is also the intersection of $\bar{L}$ and the dense set generated by the vectors $(2,2)$ and $(2,4)$. These are two representations of $L$ by maximal primary components and the two dense parts of the two intersections are different.

\section{REFERENCE}

1. B. L. van der Waerden, Moderne Algebra, vol. 2, Berlin, 1940.

Princeton University, Princeton, N. J. 